\title{
Developing an Undergraduate Laboratory in Atmospheric Physics
}

\author{
Charles Pavloski, William Brune, and George Young \\ Department of Meteorology, The Pennsylvania State University, \\ University Park, Pennsylvania
}

\begin{abstract}
Meteorological measurements and instrumentation must be part of the undergraduate education of the next generation of atmospheric scientists. These future meteorologists will be using observations from an increasingly sophisticated array of instruments and they must understand what the numbers mean. The Department of Meteorology at The Pennsylvania State University has recognized this need and has developed a required two-semester undergraduate physical meteorology sequence that couples measurements, instruments, and laboratory experiments to lecture courses in atmospheric physics. These courses help students apply concepts learned in quantitative analysis, statistics, and calculus to laboratory experiments and atmospheric observations. They also demonstrate the basic operation of several common meteorological instruments and under which conditions these measurements have meaning.
\end{abstract}

\section{Introduction}

Atmospheric science has traditionally relied upon observations, analysis, and modeling, mathematical and physical, of geophysical phenomena whose spacial and temporal scales extend from molecular to global. Regardless of the particular scale, our understanding of both specific atmospheric phenomena and of the global atmosphere as a complicated, inherently nonlinear dynamical system has been built on a foundation of observations. Historically, many of the essential measurements have been relatively simple, but manifold and geographically dispersed. Measurements today are often highly specialized and may even be unique to individual systems, such as a particular satellite or a limited network of sophisticated surface-based remote sensors (Study on Observational Systems 1991). Yet in spite of this increasing availability of diverse and high quality atmospheric measurements, most universities are poorly equipped

Corresponding author address: Charles Pavloski, Department of Meteorology, The Pennsylvania State University, 503 Walker Building, University Park, PA 16802.

E-mail:pavloski@essc.psu.edu

In final form 9 September 1994.

(C1995 American Meteorological Society to meet the challenge of educating meteorologists and atmospheric scientists about measurements.

Despite a 50-year tradition of measurements-oriented research, the Department of Meteorology at The Pennsylvania State University has, during the last 16 years, become increasingly frustrated with our apparent inability to inform undergraduates of the importance of and opportunities in measurements and technology. Fewer than $10 \%$ of our undergraduates chose to take the elective course in meteorological instrumentation. The faculty's perception, however, of the importance of these fields differs substantially from that of the students. Thus, many of the recommendations later made by the Study on Observational Systems (1991) committee were implemented by the department in the late 1980s. In particular, a revised undergraduate curriculum featuring two required measurements-oriented physical meteorology laboratory courses was introduced. We discuss in this paper the details and results of this evolving program for enhancing undergraduate measurements and instrumentation skills.

\section{Pedagogical approach}

Development of such a prototypical program in measurements and technology as part of an undergraduate atmospheric physics curriculum required three steps: 1) development of a plan linked with the remainder of the meteorology curriculum, 2) establishment of a large student laboratory with state-ofthe-art instruments and computational facilities, and 3) preparation and execution of laboratory exercises. These laboratory exercises are continually being evaluated and upgraded.

\section{a. Curriculum development plan}

The overall philosophy of our curriculum change was to balance the historical strength of the undergraduate program in dynamic and synoptic meteorology with enhancements in the general areas of atmospheric physics and observations. The revised cur- 
riculum was designed to produce graduates who are capable both in the theoretical and observational aspects of the atmospheric sciences. These students should understand not only how meteorological data are collected but also the limitations these measurement techniques impose on the observations. These insights will prepare them to better analyze data and translate their observations into knowledge.

The curriculum changes begin with the addition of a required two-semester sequence in atmospheric laboratory and measurement techniques as an integral part of the physical meteorology curriculum. This integration insures that all students develop an en-

\section{The students should understand not only how meteorological data are collected but also the limitations these measurement techniques impose on the observations.}

hanced understanding of atmospheric physics and atmospheric data measurement techniques. An associated, supporting curriculum change is the addition of a required course in meteorological applications of computers for numerical and statistical analyses. This course teaches the students how to process, interpret, and analyze meteorological data.

The atmospheric physics lecture course sequence is fairly standard. It covers such topics as radiative transfer, photochemistry, remote sensing, introductory physical climatology, aerosol and cloud phenomenology, cloud microphysics, cloud chemistry, and biogeochemical cycles. The associated laboratory courses, upon which this report focuses, are designed to give students a practical introduction to instruments, measurement techniques, and data handling procedures that are relevant to these topics. The laboratory gives the students three tools for their careers. First is an understanding of the physical principles of atmospheric phenomena. Second is a working knowledge of instruments and data analysis techniques that are used in increasingly sophisticated meteorological measurements. Third is an awareness of the limitations and potential errors associated with measuring atmospheric phenomena.

These goals are accomplished with a mixture of laboratory exercises. Some involve measurements of physical processes with simple devices that mimic the behavior of research quality instruments. The physical principles of both the atmospheric phenomena and the instruments are demonstrated. Some involve measurements with sophisticated instruments. The students thus become acquainted with research quality instrumentation. Finally, some involve data analyses of measurements made by research instruments. The students learn current data analysis techniques to derive physical quantities that they have discussed in the lecture.

\section{b. Laboratory facility development}

The equipment required for these laboratory exercises is a mixture of sophisticated, state-of-the-art instrumentation and simple laboratory facilities and devices that illustrate the physical principles of more expensive instrumentation. Although some of the instrumentation already existed in the Department of Meteorology from previous laboratory courses about meteorological instrumentation, much of it was woefully out-of-date or in poor condition. As a result, much of the instrumentation, both simple and research grade, had to be either built or purchased for these laboratory exercises.

Funding for purchasing this equipment came from the National Science Foundation through their Instrumentation and Laboratory Improvement (ILI) program. Half of the funding for larger equipment came from the Department of Meteorology, the College of Earth and Mineral Sciences, and The Pennsylvania State University. The Department of Meteorology also contributed funds and the time of research assistants to buy or build a number of essential smaller pieces of equipment and supplies.

An important feature of these laboratory exercises is the extensive use of the computer. The students use personal computers to make the measurements, analyze the data, and write and print their laboratory reports. Twelve computers are used, 10 for each group of 20 students and 2 for printing the laboratory reports. Instruments with analog outputs, such as thermocouples, thermistors, anemometers, pyranometers, and capacitance pressure gauges, are read directly by the computers through analog-to-digital conversion boards. Analog-to-digital boards transform instrument signal data into digital form for use by a computer. Once the experiments are completed, the data are transferred into a spreadsheet program where they are analyzed. The students manipulate the data, produce other spreadsheets and graphs all on the desktop personal computer.

As we began developing the topics for these laboratory courses to complement the lecture courses on atmospheric physics, we made a list of required equipment. This list was the basis for our proposal to National Science Foundation (NSF) for ILI funds. This thematic development was only the prelude to the development of the actual course exercises. The objectives of each exercise were defined and a prototype of each exercise was written and tested by the 
instructors and teaching assistants. The hardware requirements were then refined, and additional equipment was either purchased or fabricated in time to be tested for the laboratory exercise. Instructors, teaching assistants, facilities assistants, and machinists shared in this development process.

\section{c. Laboratory exercises}

The laboratory exercises are handed out a few weeks before the exercise is to be performed. Each consists of three parts: a statement of objectives, an introduction that contains the theoretical background and basic concepts of the exercise, and a procedure. In the procedure are questions that are to be answered in the laboratory report. The questions are designed to help students think about the physical properties being illustrated in the laboratory and about possible problems in the experimental techniques that might be affecting the results. Once the laboratory exercises are fully refined, these exercises will be put together into a laboratory manual.

The students produce a laboratory report at the end of each laboratory exercise. This report gives us the information necessary for evaluating the students' progress and gives them practice in organizing their thoughts and putting those thoughts on paper. The reports are kept to a few pages in length and consist of a heading, a report of laboratory activities, data, and data analysis, answers to questions, and suggestions for improvements.

In the fall semester, the primary theme of the lecture course on Atmospheric Physics is radiation. The topics of the laboratory exercises generally reflect this theme. However, several of the laboratory exercises in this semester were designed to give the students a familiarity with data collection techniques involving computers and with atmospheric measurements of common parameters.

In the first laboratory exercise, the students become familiar with the computers and the programs they will need to analyze data and write their reports. They use the Windows environment to create directories, and to create and manipulate files with File Manager. They use Windows Write to produce a description of themselves and their computer skills, and then print this description with the printer. They perform a simple exercise that teaches them the basic use of a spreadsheet for data analysis and graphing. They produce this graph on the printer and include it in their report.

In laboratory 2, the students get acquainted with the analog-to-digital (A-to-D) conversion board and learn about data sampling rates and aliasing. Once they have learned the mechanics of moving between Windows and the A-to-D, the students sample the voltage signal from a small oscillator that is connected to one of the A-to-D inputs. They sample this signal at different sampling rates, which have periods both larger and smaller than the period of the oscillator, and import these data files from the raw data directory into their own. Once they have created usable data columns within Excel and have plotted the observed signals against time, they are asked to determine the shape and frequency of the waves. They are then asked to explain the phenomena of aliasing in their own words.

The next two exercises concern measurement of temperature with thermocouples and thermistors. In these exercises, the students learn about the physical principles of temperature measurement, and then use these devices to measure the temperature of a water bath. To do this, they must first calibrate the instruments against an ice bath and boiling water so that they can establish the link between the actual temperature and the voltages measured by the thermistor or thermocouple. In this process, they also get practice with taking data with the devices attached to the computer by the A-to-D board, importing data into a spreadsheet, and analyzing them.

In the next laboratory exercise, the students begin their experience with measurements of radiation. They begin with radiometers for solar and infrared flux. A research quality radiometer is set up on the roof of the Walker Building and the students are shown the operation of the instrument. The discussion centers on the information that can be learned from measurements of radiative flux in different wavelength bands. They then return to the laboratory where they are given on diskette data taken from a radiometer at Penn State's Circleville Farm site during the summer of 1991. The students then enter these data into the spreadsheet and analyze them. Particular attention is paid to the understanding of the ratios of infrared and visible fluxes between day and night. The students are asked to explain the observations in terms of the physical principles that they have studied in the lecture course and are led through these explanations with a series of questions.

The second radiation exercise concerns the use of a sun photometer to extract information about aerosol scattering from observations of the sun. The students are shown this device in operation on the roof of the Walker Building and then return to the laboratory where they analyze data also taken at Circleville in 1991. They are able to extract the extinction coefficients, using solar photometer theory, and thus learn about the characteristic of the aerosol distribution in the State College area. Because ozone absorbs in one of the channels, they also learn about the importance of considering the gaseous absorption during such an analysis.

The third radiation exercise involves the solar 
pyranometer. The importance of the cosine response of this device is stressed, and the students measure that response, using slide projectors as the light sources. The solar pyranometers are mounted to panels on small rotary tables and the measurements are made at angles on either side of normal. The students analyze the deviations of the pyranometer response with angle and consider how these deviations affect the measurement of the heat budget at the surface of the earth.

The fourth radiation exercise ties together the concepts of temperature and radiation. Infrared thermometers are used to measure the temperature of a number of objects, first in the laboratory and then outside. By using infrared radiation to measure temperature, the students are able to see how the temperature of an object and its infrared radiation are related. They also are given exposure to various infrared devices. As they make measurements in the laboratory, they are encouraged to think of the effect that the emissivity has on the measurement of temperature. Finally, outside, they are asked to measure the temperature of the atmosphere at $20^{\circ}$ angle intervals with respect to the horizon and to plot the temperature as a function of angle. On clear days, the students are amazed to measure temperatures as low as $-70^{\circ} \mathrm{C}$ at the zenith.

The final laboratory exercise concerning radiation is a study of atmospheric absorption and Beer's Law. Using a slide projector as a light source and the solar pyranometer as the detector, the students insert pieces of smoky black plexiglass between the projector and the detector, one at a time. They record the observed signal. Upon analysis, they see that the plot of the logarithm of the transmitted light is proportional to the thickness of the plexiglass between the projector and detector and are able to deduce an absorption coefficient from this line. This laboratory is a simple, but effective, demonstration of Beer's Law.

The final laboratory of the fall semester concerns the measurement of another important meteorological parameter, wind speed. Using a 14-in. wind tunnel as a variable wind source and a hot wire anemometer as the wind speed "standard," the students measure the properties of both propeller and cup anemometers. In particular, they measure the threshold and finishing wind speeds and response of the anemometers to different wind speeds. For the propeller anemometer, they also measure the response of the anemometer for different angles of the wind with respect to the propeller axis.

In the spring semester, the students primarily study the properties of water in the atmosphere. They perform experiments involving the freezing of cloud droplets and the formation of clouds. They study the instruments used to make these measurements and analyze potential errors. They also learn proper techniques for measuring the acidity of rain.

The first two laboratory exercises concern techniques of laboratory measurements. In the first exercise, the students build a simple environmental chamber consisting of a light bulb in a base, a thick-walled cardboard tube that is larger than the bulb, a piece of foam that encloses the top of the tube, and the thermocouple. The students can control the light bulb with either a dimmer switch or an on/off switch. They manipulate the switches to maintain a preselected temperature, thus learning about proportional feedback control. They also measure the response and decay time of the chamber.

The second exercise concerns two analyses that are essential for assessing the uncertainty in an experiment. The first is propagation of error. The second is the determination of significance of the differences in two measurements. This exercise is the only one with a significant lecture, which is about one hour long. The students are told about these two issues and are given examples and solutions. They are then asked to propagate the uncertainty and analyze the significance of the differences of their measurements for a number of experiments that they have done in the first semester. In the future, these exercises will be placed earlier in the two course sequence, since proper error analysis is critical in experimental science. These techniques are then used in the analyses for the rest of the semester.

The next two laboratory exercises involve measurement of humidity. In the first exercise, the students learn to use the sling psychrometer for measurements of humidity. They are shown the technique used by operational forecasters, and then measure the vapor pressure both inside the laboratory and outside. They are asked to convert vapor pressure to relative humidity and dewpoint and to compare the outside numbers to the inside numbers. Finally, they average the measurements for the entire class and compare their results to that from a chilled mirror dewpoint hygrometer.

The second laboratory exercise on humidity involves the use of simple, chilled mirror devices to measure dewpoint. This exercise merges the concepts taught in previous exercises. The simple device consists of a covered beaker with dry ice, a brass metal strip, 6 in long and shaped like an "L," and the thermocouple. The long section of the $L$ is pushed into the beaker in contact with the dry ice, and the thermocouple is taped to the inside of the $L$ just below the turn, and just on the other side of the metal from a mirrored metal surface that has been exposed into place. The device cools from the dry ice, and the 


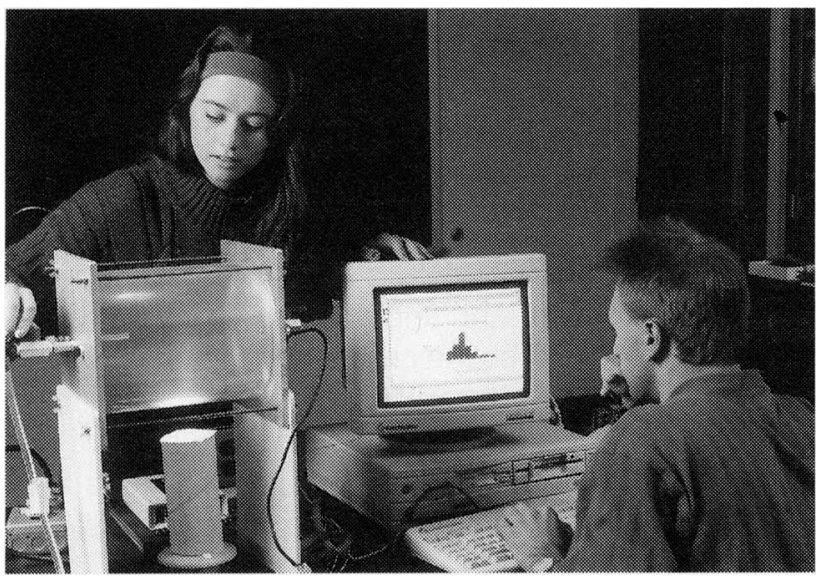

FIG. 1. Students measuring cloud droplet distribution. The size distribution that is displayed on the computer screen is the result of subsequent analysis.

mirror then collects dew (or frost) as it cools below the dewpoint. The student can hold the brass strip on the bend and warm the mirror. By alternately holding and releasing the brass strip, the student can control the dew formation on the mirror. The temperature is registered as the dew progresses across the mirror. This way, the student acts identically to the control unit of a dewpoint hygrometer. Once again, the students compare their results to that of the research grade dewpoint hygrometer.

The next laboratory exercise involves the determination of the latent heat of evaporation. The students do this by measuring the change in temperature of a heated block of aluminum as a known amount of water on that block evaporates. They are shown that evaporation alone is not cooling the block, and that they must do a control experiment to derive the result. They see that they must also account for the specific heat of the water. They assume that the specific heat of the aluminum is a standard. They then use the techniques of propagation of error and determination of significant differences to see how well they did compared to the known value.

Another exercise involves the supercooling and freezing of water drops on a cold surface. The students place several drops with diameters ranging from 0.02 to $0.8 \mathrm{~cm}$ on a cold, plastic surface and measure the temperature of that surface as it is cooled to below the freezing point. The supercooling and the temperature at which each cooled drop freezes is noted, and the drop volume is then plotted as a function of the freezing temperature. The expected result, in which the larger drops freeze first because they contain more ice nuclei, is somewhat difficult to achieve unambiguously. This difficulty results from the differences in heat transfer out of the larger drops compared to the smaller drops. Part of the purpose of this experiment is to show the students how they must be careful about many aspects of making such measurements.

The next set of exercises involves clouds. Small cloud chambers have been developed that are made of plexiglas tubing, $40-\mathrm{cm}$ diameter and $45 \mathrm{~cm}$ long, capped with "o" rings on both ends by aluminum plates. Each plate has tapped holes for vacuum and air connections, pressure and temperature sensors, and other sampling systems. The chambers are set above a light, which helps the observations of cloud formation. In the first exercise, the students become familiar with the chamber. They evacuate the chamber with a small vacuum pump and watch the clouds form. They add condensation nuclei and observe the change in the character of the cloud. They measure the adiabatic expansion of the air.

In the second exercise, the students sample the aerosols that are created in the cloud chamber (Fig. 1). A sampling system has been built that consists of a metal tube with a horn-shaped entrance, a rod that slides into the middle of this tube, but does not touch the end, a plastic disk, covered with carbon black that is attached to the end of the rod, and a syringe that goes over the other end of the other rod. This impactor is then placed into the cloud chamber. The studentsmake the cloud and pull out the syringe with a rapid motion, causing the particles to impact on the carbon black surface. They then take these plastic disks to a microscope where they can count the number of impacts and measure the diameters. From these measurements they derive the distribution function for the particles, the number density, and the total liquid water content.

The last exercise involves the measurement of the $\mathrm{pH}$ of rain and other liquids. The students are first instructed in the proper techniques for measuring $\mathrm{pH}$. They then measure several samples and standards, and their results are compared.

These are the laboratory exercises that we have done in the last few years. A few unsuccessful exercises have not been discussed here, as they will probably never be used again. We continue to develop new exercises, however, both to give the students some experience in other areas of the atmospheric sciences and to improve the concepts that we have already developed. For instance, in this next year, we will add exercises to look at ice crystal structure and growth and to measure the earth's electric field. This process of improving the exercises will be ongoing for the next several years.

\section{Course history and future}

These laboratory courses and their associated 
lecture courses on atmospheric physics have been taught for three years. One hundred and twenty-three students have taken these courses in that time. The course each semester has been taught by a faculty member and two teaching assistants, who are graduate students. This departmental commitment to this new course has made it as successful as it has been.

Overall, the comments from the students on the student evaluations have been generally positive. The students liked that the laboratory exercises often highlighted topics being discussed in the lecture courses. Students enjoyed most taking the data in the laboratory themselves. Many were also glad to get the opportunity to learn new computing skills.

The laboratory exercises have not been without problems, however. Laboratory exercises have often run longer than the two and one-half hours allotted. The instructions have not always been concise and clear, and the students sometimes did not know why they were following some procedures. Problems with using the data collection and spreadsheet analysis programs have been frequent, but seem to be tapering off as each year passes. The students preferred working with the data they took, as opposed to that given to them.

From our perspective, these courses were effective in giving the students the chance to apply concepts learned in statistics and calculus to laboratory experiments and atmospheric observations. It also showed them the basic operation of a number of common meteorological instruments, and under which conditions those measurements had meaning. These were our primary goals for this course, and to a large extent, they have been met.

However, much remains to be done. The laboratory exercises still need to be better matched with the class lectures. This match can never be perfect, because several of the exercises involved instruments that are not discussed in lecture. We have often overestimated the technical abilities of the students to make the exercises work correctly and get a reasonable result. This problem can be alleviated by improving the instructions to account for the students' skills. We are perhaps most disappointed that the students did not develop at the beginning of each laboratory exercise an idea of what the data should look like. Often, they would come to the analysis of the data with little time left and have data that were either of poor quality or entirely wrong! Thus, the instructions need to have frequent questions to get the students to think ahead. In the few laboratory exercises in which such leading questions have been included, the students knew better when they were having trouble. We will revise the remainder of the exercises accordingly.

We have found that the students gain ability best by using the tools themselves after a few instructions than by using tutorials or listening to more extensive lectures. For instance, we tried in the first year to teach the students how to use Excel with the tutorial that comes with Excel. But the students only really learned how to use Excel after they had performed a few exercises.

Students often failed to see the connection between one exercise and another. They often failed to remember techniques they had performed either with the computer software or the laboratory hardware a few exercises previously. This lack of continuity in their thinking suggests that more leading questions are necessary in the instructions, and that occasional reminders of techniques are required.

On the other hand, over the last three years, we have seen an increasing number of students who are thinking ahead, questioning results, and repeating procedures when results seem strange. We are seeing more students coming into the course who are computer literate, and may in fact complain about how "outdated" our systems are. We are seeing more students discussing with each other the questions posed in the procedure and coming to some interesting conclusions. These positive signs all point to increasing success for this type of laboratory course.

We need to continue to improve the quality of the instructions and concepts of the laboratory exercises. We need to devise new exercises that cover other topics in atmospheric physics, such as ice crystal formation and atmospheric electricity. We need to continue to improve the equipment and techniques for the exercises to maximize learning and minimize unwarranted technical difficulties. It is particularly important to get the students more involved with using research quality instrumentation as one aspect of the laboratory.

Within the next year, the laboratory exercises will be collected into a laboratory exercise lab book that the students can purchase through the bookstores. Ideally, this lab book will have more exercises than can comfortably be accomplished in 30 weeks, so that the instructor and teaching assistants can choose the ones they want to perform that semester.

Acknowledgments. This work has been supported by NSF Grant USE-9151806. Professors Thomson, Ackerman, Albrecht, Curry, Lamb, and Wyngaard, and Graduate Teaching Assistants James Cohen, Robert Edsall, Brian Lambert, Patrick Marquis, Tom Salem, and Timothy Tyce contributed greatly to the success of these courses.

\section{References}

Study on Observational Systems, 1991: A review of meteorological and oceanographic education in observational techniques and the relationship to national facilities and needs. Bull. Amer. Meteor. Soc., 72, 815-826. 